\title{
Performance test of the MAlKo active target
}

\author{
$\operatorname{AUTHOR}(S):$ \\ Furuno, T.; Kawabata, T.; Ong, H.J.; Adachi, S.; \\ Ayyad, Y.; Baba, T.; Fujikawa, Y.; ... Tran, D.T.; \\ Tsumura, M.; Watanabe, H.D.
}

\section{CITATION:}

Furuno, T. ... [et al]. Performance test of the MAIKo active target. Nuclear Instruments and Methods in Physics Research Section A: Accelerators, Spectrometers, Detectors and Associated Equipment 2018, 908: 215-224

\section{ISSUE DATE:}

2018-11-11

URL:

http://hdl.handle.net/2433/234586

\section{RIGHT:}

(c) 2018. This manuscript version is made available under the CC-BY-NC-ND 4.0 license

http://creativecommons.org/licenses/by-nc-nd/4.0/; The full-text file will be made open to the public on 11 November 2020 in accordance with publisher's 'Terms and Conditions for Self-Archiving'.; This is peer-reviewed version. この論文 は査読済著者稿です。; This is not the published version. Please cite only the published version.; この論文は出版社版で ありません。引用の際には出版社版をご確認ご利用ください。 


\title{
Performance test of the MAIKo active target
}

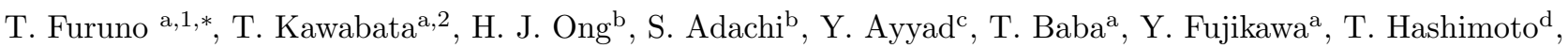

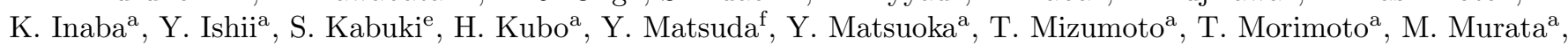

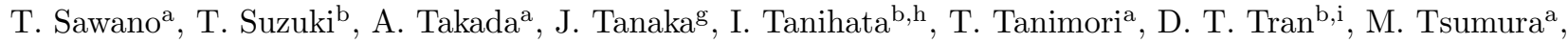
H. D. Watanabe ${ }^{\mathrm{a}}$

\author{
${ }^{a}$ Department of Physics, Kyoto University, Kitashirakawa Oiwake, Sakyo, Kyoto 606-8502, Japan \\ ${ }^{b}$ Research Center for Nuclear Physics, Osaka University, 10-1 Mihogaoka, Ibaraki, Osaka 567-0047, Japan \\ ${ }^{c}$ Lawrence Berkeley National Laboratory, 1 Cyclotron Road, Berkeley, CA 94720, USA \\ ${ }^{d}$ Rare Isotope Science Project, Institute for Basic Science, Yuseong-daero 1689-gil, Yuseong-gu, Daejeon 305-811, Korea \\ ${ }^{e}$ School of Medicine, Tokai University, 143 Shimokasuya, Isehara, Kanagawa 259-1193, Japan \\ ${ }^{f}$ Cyclotron and Radioisotope Center, Tohoku University, 6-3 Aoba Aramaki, Aoba, Sendai, Miyagi 980-8578, Japan \\ ${ }^{g}$ Institut für Kernphysik, Technische Universität Darmstadt, Karolinenplatz 5, 64289 Darmstadt, Germany \\ ${ }^{h}$ International Research Center for Nuclei and Particles in the Cosmos, and School of Physics and Nuclear Energy Engineering, Beihang \\ University, 37 Xueyuan Road, Haidian, Beijing 100191, China \\ ${ }^{i}$ Institute of Physics, Vietnam Academy of Science and Technology, 10 DaoTan, BaDinh, Hanoi 100000, Vietnam
}

\begin{abstract}
A new active target named MAIKo (Mu-PIC based Active target for Inverse Kinematics。) has been developed at Kyoto University and Research Center for Nuclear Physics (RCNP), Osaka University. MAIKo is suited for missing-mass spectroscopy of unstable nuclei at forward scattering angles in inverse kinematics. MAIKo consists of a time projection chamber (TPC), which incorporates a micro-pixel chamber ( $\mu$-PIC) as the electron multiplication and collection system. In MAIKo, the medium gas also plays the role of a reaction target, thus allowing detection of low-energy recoil particles with high position resolution. The MAIKo TPC was commissioned with $\mathrm{He}(93 \%)+\mathrm{iso}_{-} \mathrm{C}_{4} \mathrm{H}_{10}(7 \%)$ and $\mathrm{He}(93 \%)+\mathrm{CO}_{2}(7 \%)$ mixture gasses at $430 \mathrm{hPa}$. The gas gain and the angular resolution of MAIKo were evaluated with an alpha source and a ${ }^{4} \mathrm{He}$ beam at $56 \mathrm{MeV}$. The TPC was stably operated up to $1000-\mathrm{kcps}$ beam intensity. A tracking algorithm using the Hough transform method has been developed to analyze scattering events. An angular resolution of $1.3^{\circ}$ was achieved for scattered ${ }^{4} \mathrm{He}$ particles.
\end{abstract}

Keywords: Active target, MAIKo, Time projection chamber, $\mu$-PIC, Missing-mass spectroscopy, Hough transformation

\section{Introduction}

Direct reactions with light ions are useful probes to investigate the structures of both stable and unstable nuclei owing to the relative simplicity of reaction mechanisms 5 [1. The direct reactions are dominant processes at beam energy of several tens $\mathrm{MeV} / \mathrm{u}$. Various reactions such as ${ }_{20}$ elastic and inelastic scattering, charge exchange, transfer and knock-out reactions have been widely employed for spectroscopic studies of stable nuclei mostly with $p, d$, ${ }_{10}{ }^{3} \mathrm{He}$, and ${ }^{4} \mathrm{He}$ beams. In these reactions, measurements at forward angles in the center-of-mass (CM) frame are ${ }_{25}$ especially important because the reaction mechanism is simple and the experimental results are less ambiguously

\footnotetext{
${ }^{*}$ Corresponding author

Email address: furuno@rcnp.osaka-u.ac.jp (T. Furuno )

${ }^{1}$ Present address: Research Center for Nuclear Physics, Osaka University, 10-1 Mihogaoka, Ibaraki, Osaka 567-0047, Japan. Tel.: +81-6-6879-8857; Fax: +81-6-6879-8899.

${ }^{2}$ Present address: Department of Physics, Osaka University, 1-1 Machikaneyama, Toyonaka, Osaka 560-0043, Japan
}

interpreted. With the recent developments of new facilities 5 5, measurements of direct reactions have been extended away from the stability line. These experiments have been performed under the inverse kinematic conditions where hydrogen or helium target is bombarded with RI beams. There are two methods to determine the excitation energy of the unstable nuclei: the invariant-mass spectroscopy and the missing-mass spectroscopy.

The invariant-mass spectroscopy has been widely applied in many earlier experiments using relatively highenergy (> $50 \mathrm{MeV} /$ nucleon) RI beams, to obtain the excitation-energy spectra of unstable nuclei by detecting all fragments emitted from the beam particles. This method enables usage of a thick liquid or solid target which ensures the highest yield at around zero degree. However, the application of this technique is limited to measurements in which multiplicity of the fragments is low.

On the other hand, the missing-mass spectroscopy, which requires detection of only the recoil particle from the target, can be applied regardless of the multiplicity. However, 
35 measurements at forward angles in the CM frame require the detection of low-energy recoil particles. For example, the energy of the recoil alpha particle is as low as $0.5 \mathrm{MeV}$ at $\theta_{\mathrm{CM}}=3^{\circ}$ in the alpha inelastic scattering off ${ }^{10} \mathrm{C}$ at 75 $\mathrm{MeV} / \mathrm{u}$, which is one of our first priority experiments. In 40 order to detect such low-energy recoil particles with external detectors, the target must be extremely thin, at the expense of the luminosity.

One solution to the above-mentioned problem is to store RI beams in a storage ring and use an internal gas-jet

45 target [6]. This technique allows the use of an extremely thin $\left(\sim 10 \mathrm{pg} / \mathrm{cm}^{2}\right)$ target while maintaining the necessary luminosity because the unreacted beams are injected repeatedly onto the target. Recently, measurements of proton elastic scattering and alpha inelastic scattering were 50 successfully performed 7 9. However, this technique is only applicable to nuclei whose life times are longer than a few seconds because it takes some time to store and cool RI beams in a storage ring.

The use of a time projection chamber (TPC) as an 5 active target is another solution. The essential feature of the active target is the use of the detection medium gas of the TPC as a target gas. Typically, helium, hydrogen, deuterium or hydrocarbon gas is used as the detection medium gas. The TPC enables three-dimensional reconstruction of charged-particle trajectories. Since the reaction occurs inside the sensitive volume of the TPC, the detection threshold for the recoil particles can be lowered and the solid angle for the recoil particles is increased up to almost $4 \pi$. Moreover, the luminosity can be increased by extending the length of a TPC along the beam axis while maintaining a low detection threshold. The reconstructed trajectory of a recoil particle determines the recoil angle and recoil energy, which are used to calculate the missing mass. Several active target systems have been 70 developed 10 16, and measurements using these detectors have been reported [17 24. A comprehensive review on the recent developments of active targets is given in Ref. [25].

Recently, a new active target named MAIKo (Mu-PIC 75 based Active target for Inverse Kinematics ${ }_{\circ}$ ) was jointly developed by Kyoto University and Research Center for Nuclear Physics (RCNP), Osaka University to perform missing-mass spectroscopies using RI beams at several tens $\mathrm{MeV} /$ nucleon at the exotic nuclei (EN) beam line at RCNP 26, 27. We adopt a strip-type readout instead of a padtype readout to achieves the finest readout pitch among the existing active targets while keeping the number of readout channel small. The gas gain and the angular resolution of MAIKo were evaluated by using an alpha source and a ${ }^{4} \mathrm{He}$ beam at $56 \mathrm{MeV}$.

The paper is organized as follows. The design of the MAIKo active target is described in Section 2, Measurements using an alpha source are reported in Section 3 followed by results of the performance test using a ${ }^{4} \mathrm{He}$ beam

90 in Section 4. The analysis of scattering events is discussed in Section 5. The summary and the future outlook are given in Section 6.

\section{Design of MAIKo}

\subsection{Overview}

A schematic view of the MAIKo system is drawn in Fig. 1. The field cage of the MAIKo TPC has a volume of $150 \times 150 \times 140 \mathrm{~mm}^{3}$. The TPC is installed in a stainlesssteel vacuum chamber with a volume of about $30 \mathrm{~L}$. The chamber is filled with helium gas, which works as the ${ }^{4} \mathrm{He}$ target as well as the detector gas, with a small fraction of quenching gas. The pressure of the detector gas can be changed from $100 \mathrm{hPa}$ to $2000 \mathrm{hPa}$ according to the experimental purpose.

The detector gas is ionized by charged particles, and the ionization electrons are drifted vertically downwards along the electric field formed by the TPC field cage. These electrons are multiplied and detected with the micro-pixel chamber ( $\mu$-PIC) [28. The $\mu$-PIC is a micro-pattern gaseous detectors developed at Kyoto University. The $\mu$-PIC has been successfully employed in a Compton camera for gamma ray imaging 2932 , in the dark matter search experiment 33. 34, and in neutron imaging 35, 36. The sensitive area of the $\mu$-PIC is $102.4 \times 102.4 \mathrm{~mm}^{2}$, which limits the sensitive volume of the TPC to $102.4 \times 102.4 \times 140 \mathrm{~mm}^{3}$. Details of the $\mu$-PIC are described in Section 2.3

High-energy recoil particles which punch through the sensitive volume of the TPC are detected by ancillary detectors consisting of silicon ( $\mathrm{Si}$ ) detectors and thalliumdoped cesium iodide $[\mathrm{CsI}(\mathrm{Tl})]$ scintillators, which are described in Section 2.5

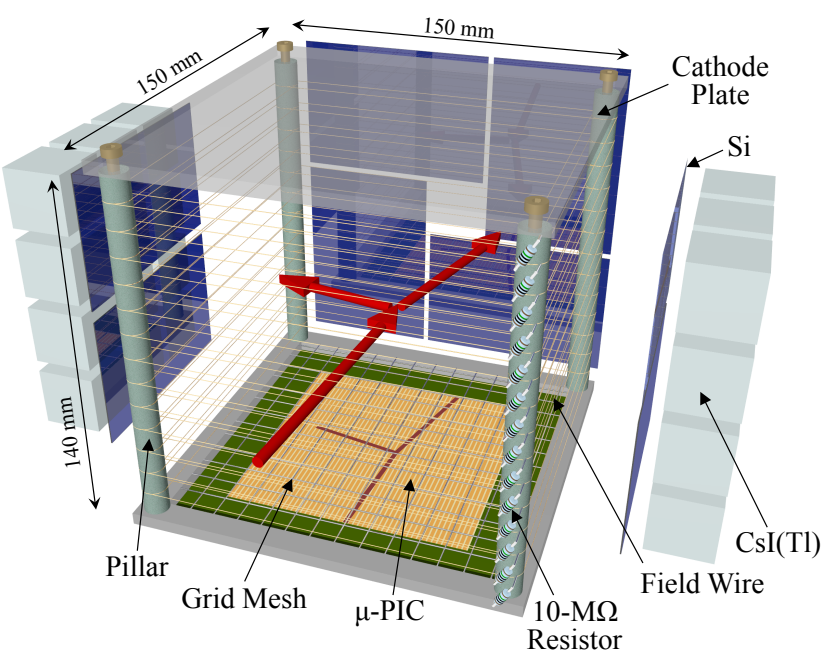

Figure 1: Schematic view of the MAIKo system.

\subsection{Field cage}

The electric circuit of the field cage is shown in Fig. 2. The field cage forms a homogeneous vertical electric field inside the sensitive volume of the TPC. The field-cage 
cathode is made of a 5-mm-thick aluminum plate. The ${ }_{16}$ size of the cathode plate is $150 \times 150 \mathrm{~mm}^{2}$. The ionization electrons are drifted towards a nickel grid mesh, which is placed $140 \mathrm{~mm}$ below the cathode plate. The diameter of the mesh wire is $150 \mu \mathrm{m}$ and the pitch size is glass-reinforced epoxy (G10) frame. The electric potential of the grid is tuned so that the grid is transparent to the drift electrons but opaque for the positive ions generated by the avalanche on the $\mu$-PIC. When the gas pressure

135 is $430 \mathrm{hPa}$, negative high voltages of $-3600 \mathrm{~V}$ and $-800_{170}$ $\mathrm{V}$ are typically applied to the cathode plate and the grid mesh, respectively, thus generating an electric field of 200 $\mathrm{V} / \mathrm{cm}$ in the field cage. The ion back flow ratio is estimated to be less than $2 \%$ using a simulation with the $\mathrm{mm}$ are mounted between the cathode plate and the grid mesh to sustain the field-cage structure as well as to hold the field wires. The drift field is uniformized by $13 \mathrm{Be}-\mathrm{Cu}$ wires winding doubly around the G10 pillars at $10-\mathrm{mm}$ inthey are connected via a $10-\mathrm{M} \Omega$ metal-film resistor chain to make a voltage divider. The tension of the wires is kept at $3 \mathrm{~N}$

A finite element calculation with the computer code neBEM [38] was performed to check the uniformity of the electric field. The neBEM code is implemented in the Garfield code. According to the simulation, the field cage ensures that the distortion of the electric field is kept within $2 \%$ in the sensitive volume even when the Si detectors are grounded and installed at $30 \mathrm{~mm}$ away from the field cage.

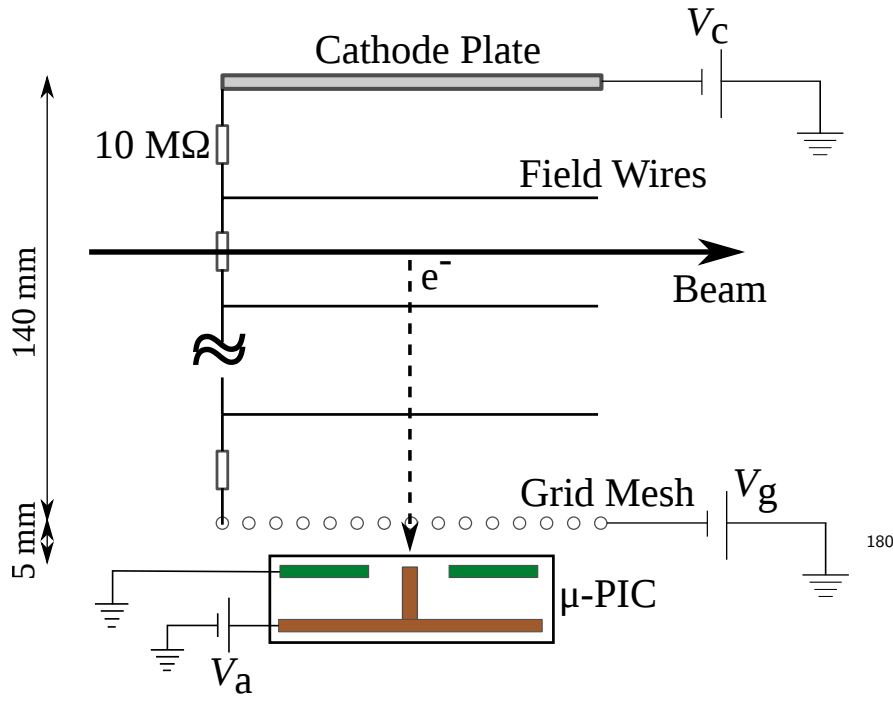

Figure 2: Electric circuit of the field cage.

\subsection{Micro-pixel chamber $(\mu-P I C)$}

The drifted electrons are multiplied by the $\mu$-PIC placed $5 \mathrm{~mm}$ below the grid mesh. A schematic structure of the
$\mu$-PIC is shown in Fig. 3 The $\mu$-PIC is fabricated using a printing circuit board (PCB) technology. The anode and cathode electrodes are printed on both sides of a 100- $\mu \mathrm{m}$ thick polyimide base. The cathode strips of 314- $\mu \mathrm{m}$ width are printed on the front side of the base with a pitch of 400 $\mu \mathrm{m}$. These strips have holes of $256-\mu \mathrm{m}$ diameter with $400-$ $\mu \mathrm{m}$ spacing along the strips. At the center of the holes, anode pixels are printed. The diameter of the anode pixels is $50 \mu \mathrm{m}$. The anode pixels are extended to the back side of the base and connected to the anode strips which are orthogonal to the cathode strips. The cathode strips are grounded while positive high voltage is applied to the anode strips which forms an electric field around the anode pixels strong enough to induce an electron avalanche. Typical values of the high voltage are from 300 to $400 \mathrm{~V}$.

Each strip of the anode and cathode is connected to a readout circuit to provide a two-dimensional fine image of the ionization electrons. The $\mu$-PIC consists of the 256 anode strips and the 256 cathode strips which determine the sensitive area of $102.4 \times 102.4 \mathrm{~mm}^{2}$.

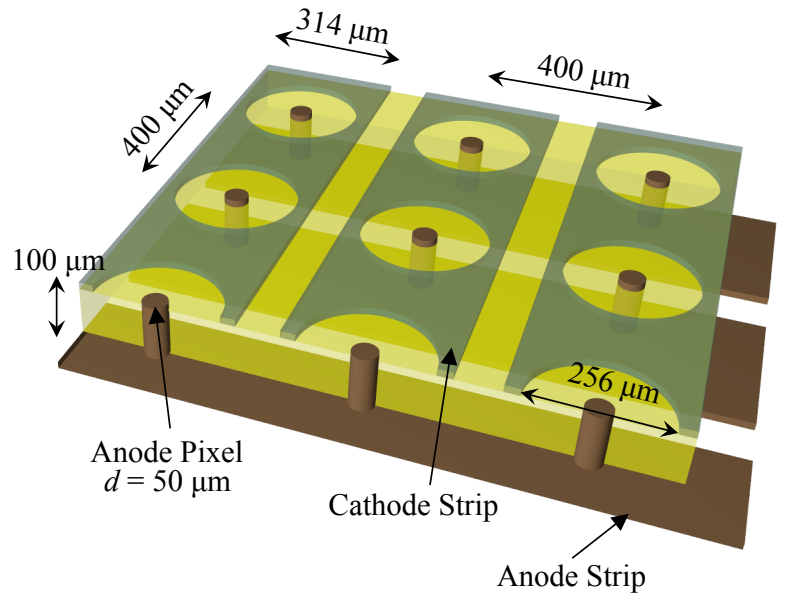

Figure 3: Schematic structure of the $\mu$-PIC 28. The $\mu$-PIC consists of anode pixels and cathode strips fabricated with a pitch of 400 $\mu \mathrm{m}$. Positive high voltage is applied to the anode pixels while the cathode strips are grounded. The drifted electrons form avalanches around the anode pixels. Signals induced by the electron avalanches are read out by the anode and cathode strips which are orthogonally arranged.

\subsection{Readout system}

The anode and cathode strips are connected to the capacitor and resistor $(\mathrm{CR})$ circuit boards. The CR circuit boards consist of 1-G $\Omega$ resistors for the high voltage bias supply and $100-\mathrm{pF}$ capacitors for the coupling to the preamplifiers. Figure 4 shows a photograph of the CR circuit boards mounted on top of the TPC vacuum chamber. The TPC field cage is installed upside-down in the vacuum chamber. The PCB board of the $\mu$-PIC is mounted to the lid of the chamber, and the CR circuit boards are directly connected to the PCB board. This CR circuit 
boards are used also for the vacuum feed-through for the 225 $\mu$-PIC signals.

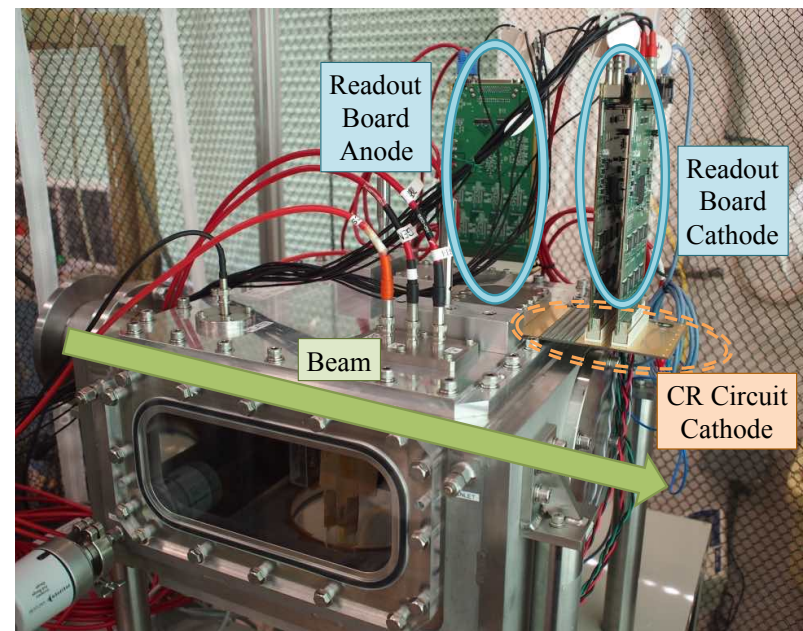

Figure 4: Photograph of the TPC vacuum chamber and the readout boards. The CR circuit board for the cathode strips is circled by the orange-dashed lines and the readout boards are circled by the bluesolid lines. The direction of the beam axis is shown by the green arrow.

Analog signals from the $\mu$-PIC are processed by the readout electronics boards specially developed by the cosmicray group at Kyoto University [39]. Each board processes 128 channels. In MAIKo, four boards are used (two for the 256 anode strips and two for the 256 cathode strips). A block diagram displaying the signal processing in the readout boards is shown in Fig. 5 .

Each readout board consists of eight application specific integrated circuit (ASIC) chips named FE2009bal 39. and a field programmable gate array (FPGA) chip. In each ${ }_{245}$ ASIC chip, analog signals from 16 adjacent electrodes of the $\mu$-PIC are amplified, shaped and discriminated. The input range of the FE2009bal chips is $\pm 1 \mathrm{pC}$ and the gain of the amplifier is $0.8 \mathrm{~V} / \mathrm{pC}$. The threshold levels of the 128-channel discriminators are individually adjusted via the SiTCP communication [40].

The discriminated signals of the 128 channels are transthe FPGA chip. The transferred signals are then synchronized with a $100-\mathrm{MHz}$ clock of the FPGA chip. The timing of the discriminated signals provides the electron drift time, and the signal duration (time over threshold: TOT) is approximately proportional to the charge collected by the $\mu$-PIC. In this paper, the first clock and the last clock of the TOT is denoted as the leading edge and the trailing edge, respectively. The analog outputs from the FE2009bal chips are summed with adjacent 32 strips and digitized by 8-bit, 25-MHz flash-ADCs (FADC) signals are also split and transmitted via the LEMO connectors on the readout boards to be used to make a TPC self trigger. The noise level is typically $\pm 5 \mathrm{mV}$. The dynamic range of the FE2009bal chip is $\pm 0.8 \mathrm{~V}$. During the

measurements using the ${ }^{4} \mathrm{He}$ beam, we adjusted the gas gain of the $\mu$-PIC so that the pulse height of beam particles is $30 \mathrm{mV}$, which is high enough to discriminate signals from noise. Under such conditions, the TPC can measure the energy loss of the particles up to about 25 times larger than that of the beam particles. The discriminated 128ch hit pattern and the 4-ch FADC data are continuously stored in a ring buffer in the FPGA chip for $10.24 \mu \mathrm{s}$. Upon receiving an external trigger signal, the data stored in the ring buffer is written out to two VME memory modules af35 ter the data formatting in the FPGA chip. The TPC data is acquired on an event-by-event basis together with the data from the ancillary detectors and the beam-line detectors via the VME bus using the data acquisition (DAQ) program developed at RIBF [41. The trigger signal is usually generated by the $\mathrm{Si}$ detectors or by discriminating the analog signals from the $\mu$-PIC strips transmitted via the LEMO connectors on the readout boards.

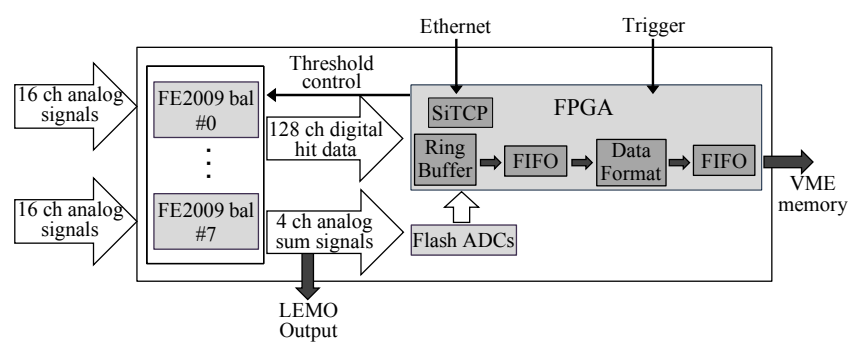

Figure 5: Block diagram of the signal processing in the readout electronics board 39].

As shown in Fig. 6, the anode and cathode strip numbers and the electron drift time in each strip provide the two-dimensional projections of the trajectories. The threedimensional track is reconstructed by combining the anode and cathode projections.

\subsection{Ancillary detectors}

High-energy recoil particles which punch-through the TPC are detected with Si detectors followed by $\mathrm{CsI}(\mathrm{Tl})$ crystals. The Si detectors have a sensitive area of $90 \times$ $60 \mathrm{~mm}^{2}$ with a thickness of $325 \mu \mathrm{m}$ or $500 \mu \mathrm{m}$ depending on experimental requirements. The $\mathrm{CsI}(\mathrm{Tl})$ crystals have a volume of $30 \times 30 \times 30 \mathrm{~mm}^{3}$. They are wrapped by enhanced specular reflector (ESR) films from 3M 42, and their back side is attached to Si-PIN photodiodes with which the scintillation photons are detected.

Two Si detectors are installed at the left and the right sides of the TPC followed by $\mathrm{CsI}(\mathrm{Tl})$ detectors. Four $\mathrm{Si}$ detectors are installed at downstream of the TPC. As illustrated in Fig. 1] these four Si detectors are arranged to form a $30-\mathrm{mm}$ square hole at the beam position to allow the beam particles to pass through.

The $\mathrm{Si}$ and $\mathrm{CsI}(\mathrm{Tl})$ detectors are placed close to the TPC field cage at a distance which is safe enough to avoid the discharge from the field cage to the Si detectors. 


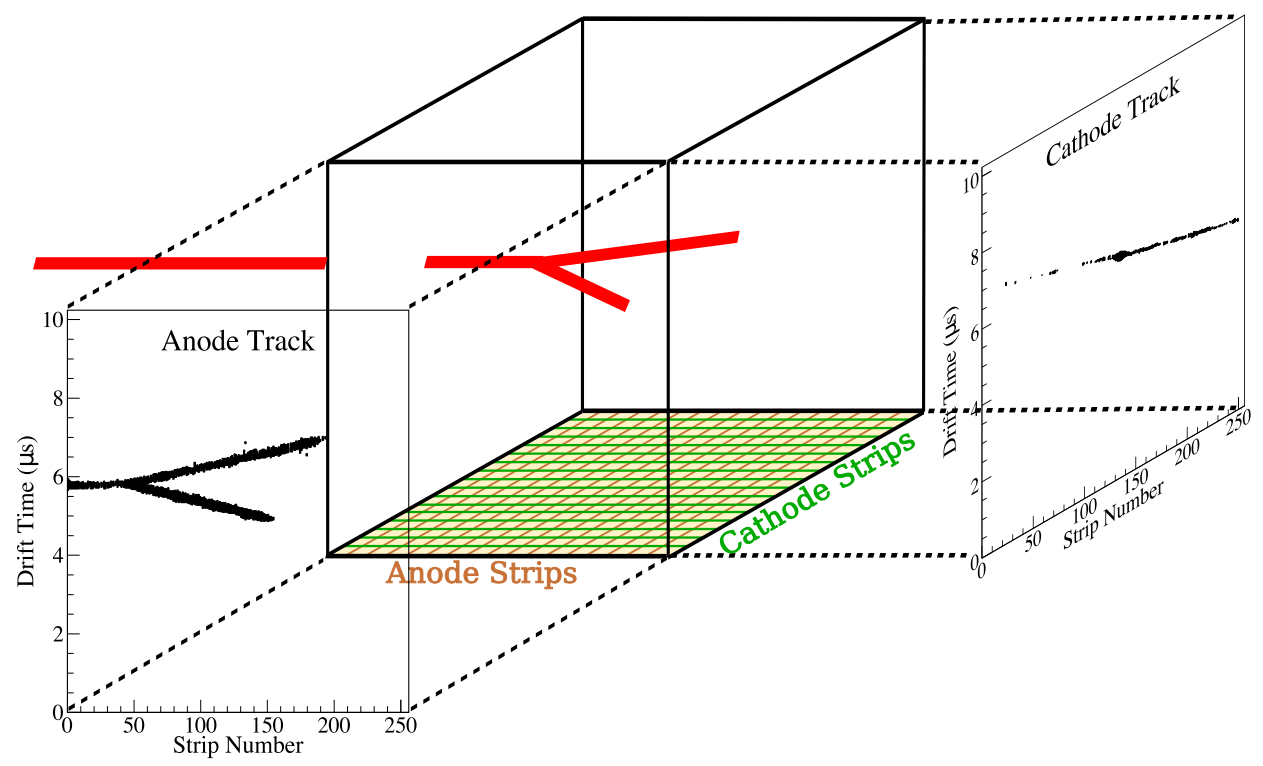

Figure 6: Reconstruction of a track from the TPC data. The anode and cathode strip numbers and the electron drift time in each strip give the two-dimensional projections of the particle trajectories.

\section{Measurement of the gas gain of the $\mu$-PIC with 300 an alpha source}

The MAIKo TPC was commissioned using an ${ }^{241} \mathrm{Am}$ alpha source to measure the gas gain of the $\mu$-PIC. The TPC chamber was filled with $\mathrm{He}(93 \%)+$ iso- $_{4} \mathrm{H}_{10}(7 \%)$ mixture gas at $430 \mathrm{hPa}$. The alpha source was mounted upstream of the TPC. The distance between the alpha source and the sensitive volume of the TPC was $61 \mathrm{~mm}$. The range of the $5.48-\mathrm{MeV}$ alpha particles from ${ }^{241} \mathrm{Am}$ in the detector gas is $264 \mathrm{~mm}$ according to the calculation using the SRIM code 43. Since this range is long enough for the alpha particles to penetrate the sensitive volume of the $\mathrm{TPC}$, these alpha particles were detected by one of the $\mathrm{Si}$ detectors installed downstream of the TPC. Signals from the Si detectors were used to trigger the DAQ system of the TPC. Measurements were performed for different bias voltages of the grid mesh and the anode of the $\mu$-PIC. The electric field between the cathode plate and the grid mesh was kept at $200 \mathrm{~V} / \mathrm{cm}$.

A typical analog signal from 32 anode strips obtained by the FADC is shown in Fig. 7. The flight direction of the alpha particles was almost perpendicular to the anode strips. The charge $Q_{1}$ collected by the anode strips after electron avalanche was derived by integrating the FADC pulse. The initial charge $Q_{0}$ caused by the alpha particle is given by $Q_{0}=e\left(\Delta E_{\mathrm{He}} / W_{\mathrm{He}}+\Delta E_{\mathrm{iC}_{4} \mathrm{H}_{10}} / W_{\mathrm{iC}_{4} \mathrm{H}_{10}}\right) . \Delta E_{\mathrm{He}}$ and $\Delta E_{\mathrm{iC}_{4} \mathrm{H}_{10}}$ are the energy losses of the alpha particles along the passage of $12.8 \mathrm{~mm}$ above the anode strips in the helium and iso-butane gases at each partial pressure. $e$ is the charge of an electron. The $\Delta E_{\mathrm{He}}$ and $\Delta E_{\mathrm{iC}_{4} \mathrm{H}_{10}}$ are estimated to be $85 \mathrm{keV}$ and $103 \mathrm{keV}$ by the SRIM code. $W_{\mathrm{He}}(41 \mathrm{eV})$ and $W_{\mathrm{iC}_{4} \mathrm{H}_{10}}(26 \mathrm{eV})$ are the mean ionization energies of the $\mathrm{He}$ and iso- $\mathrm{C}_{4} \mathrm{H}_{10}$ gasses, respectively [44].
The gas gain was determined from $Q_{1} / Q_{0}$.

Figure 8 shows the measured gas gains with different voltages applied to the grid mesh as a function of the bias voltage applied to the anode strips of the $\mu$-PIC. The gas gain increases with the voltage applied to the grid mesh. This is because the electric field around the anode of the $\mu$-PIC becomes stronger with increased grid voltage. The maximum gain $(\sim 3000)$ was limited by the discharge of the $\mu$-PIC.

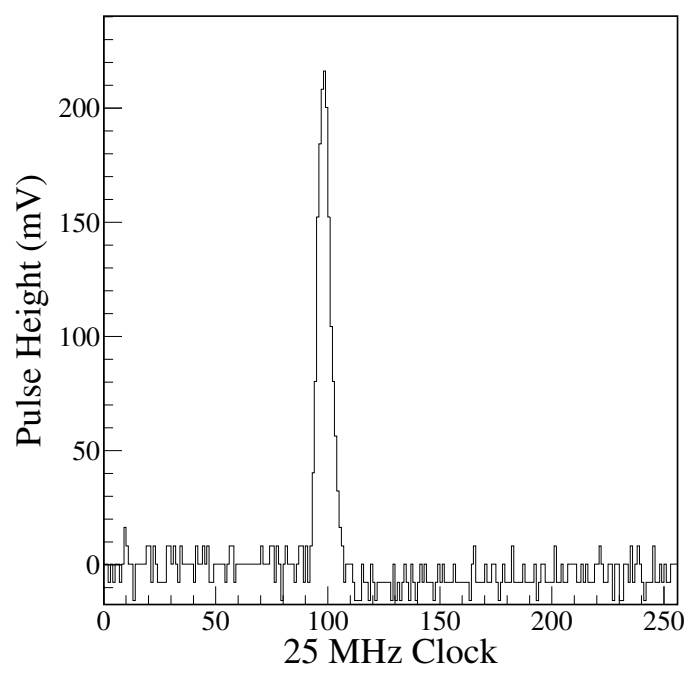

Figure 7: Typical signal acquired with the FADC for an alpha particle emitted from an ${ }^{241} \mathrm{Am}$ alpha source. The signal was summed over 32 consecutive anode strips on the $\mu$-PIC which have a total width of $12.8 \mathrm{~mm}$ 


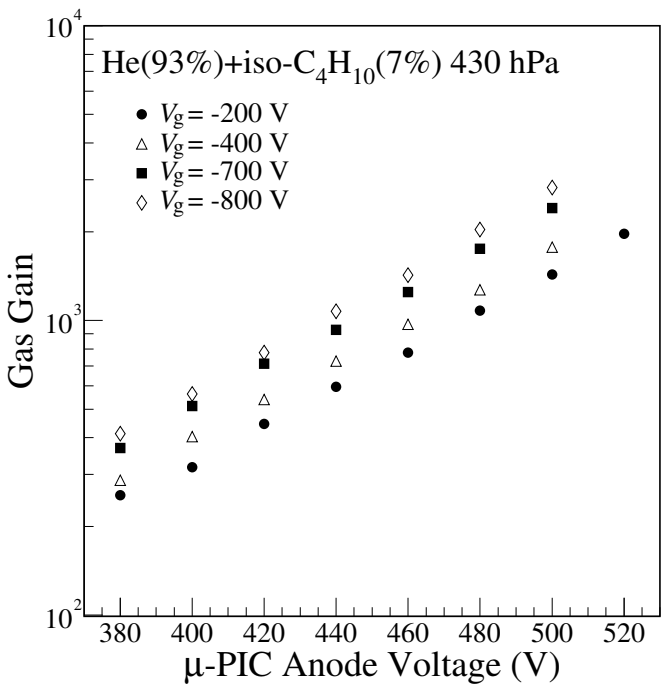

Figure 8: Gas gain of the $\mu$-PIC as a function of the $\mu$-PIC anode voltage. The gain was measured with a $\mathrm{He}(93 \%)+$ iso- $_{4} \mathrm{H}_{10}(7 \%)$ mixture gas at $430 \mathrm{hPa}$. The measurement was performed with different voltages applied to the grid mesh: $-200 \mathrm{~V}$ (solid circles), ${ }^{340}$ $-400 \mathrm{~V}$ (open triangles), $-700 \mathrm{~V}$ (solid squares), and $-800 \mathrm{~V}$ (open diamonds).

\section{Measurements with a ${ }^{4} \mathrm{He}$ beam}

\subsection{Experimental setup}

A test experiment was carried out using a $56-\mathrm{MeV}^{4} \mathrm{He}$ beam from the azimuthally varying field (AVF) cyclotron at RCNP to study the detector performances under high counting rates and to acquire scattering events.

The experimental setup is shown in Fig. 9. The MAIKo system was installed at the F2 focal plane of the RCNP EN beam line. The MAIKo chamber was filled with $\mathrm{He}(93 \%)$ + iso- $\mathrm{C}_{4} \mathrm{H}_{10}(7 \%)$ or $\mathrm{He}(93 \%)+\mathrm{CO}_{2}(7 \%)$ mixture gas at $430 \mathrm{hPa}$. The chamber was separated from the vacuum section of the beam line by a $25-\mu$ m-thick aramid window film. The diameter of the window film was $90 \mathrm{~mm}$. The four $500-\mu$ m-thick Si detectors (left and right sides) and the four 325- $\mu \mathrm{m}$-thick Si detectors (downstream) were installed 100-mm away from the center of the TPC sensitive volume as described in Section 2.5. The four Si detectors at the left and right sides were used to create a trigger signal for the scattering events. A plastic scintillator with a thickness of $200 \mu \mathrm{m}$ was installed upstream of the $\mathrm{TPC}_{3}$ to measure the beam intensity. This scintillator was also used to define the start timing of the TPC. The energy losses of the ${ }^{4} \mathrm{He}$ beam by the plastic scintillator and the aramid foil are $3.0 \mathrm{MeV}$ and $0.4 \mathrm{MeV}$, respectively. The energy and angular stragglings in the two materials are 0.1 $\mathrm{MeV}$ and $0.3 \mathrm{deg}$, respectively, which are negligible in the present analysis.

\subsection{Angular resolution}

The angular resolution of the TPC was evaluated from $_{365}$ the ${ }^{4} \mathrm{He}$ beam events. The data was taken with both $\mathrm{He}+$

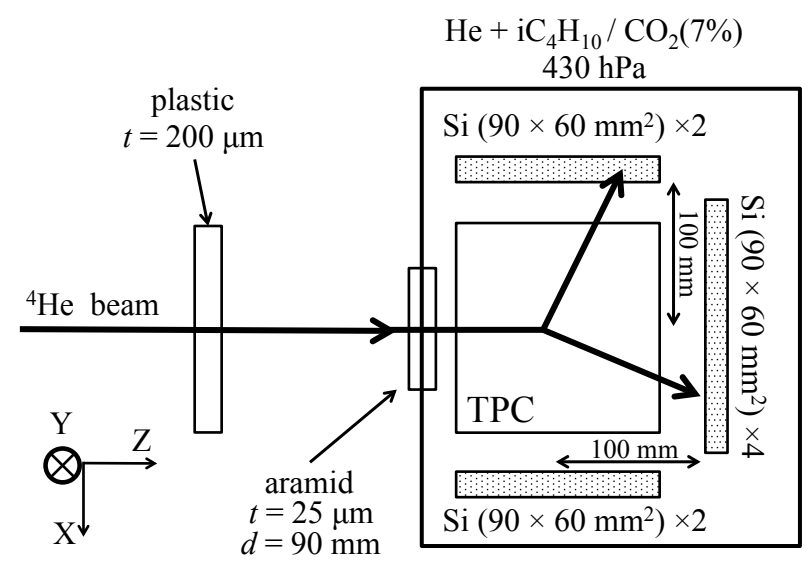

Figure 9: Top view of the experimental setup at the RCNP EN beam line.

iso- $\mathrm{C}_{4} \mathrm{H}_{10}(7 \%)$ and $\mathrm{He}+\mathrm{CO}_{2}(7 \%)$ gases at $430 \mathrm{hPa}$. The voltage parameters of the TPC (cathode plate: $V_{\mathrm{c}}$, grid mesh: $V_{\mathrm{g}}$, and $\mu$-PIC anode: $V_{\mathrm{a}}$, see Fig. 2 for both of the gas mixtures are listed together with the drift velocity $v$ in Table 1. These operating voltages were chosen so as to optimize the gas gain without discharge. The drift 5 velocities were calculated with the Magboltz code [45]. We confirmed that the calculated drift velocities agreed with the measured drift velocities within $5 \%$.

Table 1: High voltages applied to the cathode plate $\left(V_{\mathrm{c}}\right)$, the grid mesh $\left(V_{\mathrm{g}}\right)$, and the anode of $\mu$-PIC $\left(V_{\mathrm{a}}\right)$ during the commission with a ${ }^{4} \mathrm{He}$ beam. The drift velocities $(v)$ with these high voltages estimated by the Magboltz simulation are also listed.

\begin{tabular}{ccccc}
\hline \hline Gas & $V_{\mathrm{c}}(\mathrm{V})$ & $V_{\mathrm{g}}(\mathrm{V})$ & $V_{\mathrm{a}}(\mathrm{V})$ & $v(\mathrm{~cm} / \mu \mathrm{s})$ \\
\hline $\mathrm{He}+\mathrm{iC}_{4} \mathrm{H}_{10}$ & -3500 & -700 & 500 & 1.59 \\
$\mathrm{He}+\mathrm{CO}_{2}$ & -3200 & -980 & 550 & 1.34 \\
\hline \hline
\end{tabular}

Figure 10 shows a typical track of a beam particle reconstructed from the anode data. The anode strips were perpendicular to the beam axis. The $Z$ coordinate was determined from the anode strip number, and the $Y$ coordinate was determined from the drift time multiplied by the drift velocity. As described in Section 2.4 the time over threshold was recorded for each strip, but only the middle clock between the leading edge and the trailing edge was used in the analysis.

To evaluate the angular resolution, the tracks of the beam particles were divided into two parts, upstream and downstream. Each part contains data for the respective 128 strips $(51.2 \mathrm{~mm})$. These tracks were fitted separately by straight lines to obtain the angles of the track $\left(\theta_{\text {up }}\right.$ and $\left.\theta_{\text {down }}\right)$. $\theta_{\text {up }}$ should agree with $\theta_{\text {down }}$ within the angular straggling of the ${ }^{4} \mathrm{He}$ beam (less than $0.05^{\circ}$ ). However, due to the limited angular resolution of the TPC, the distribution of $\theta_{\text {up }}-\theta_{\text {down }}$ is smeared out from $0^{\circ}$ as shown in Fig. 11. The standard deviation $\left(\sigma_{\text {up }- \text { down }}\right)$ of the distri- 


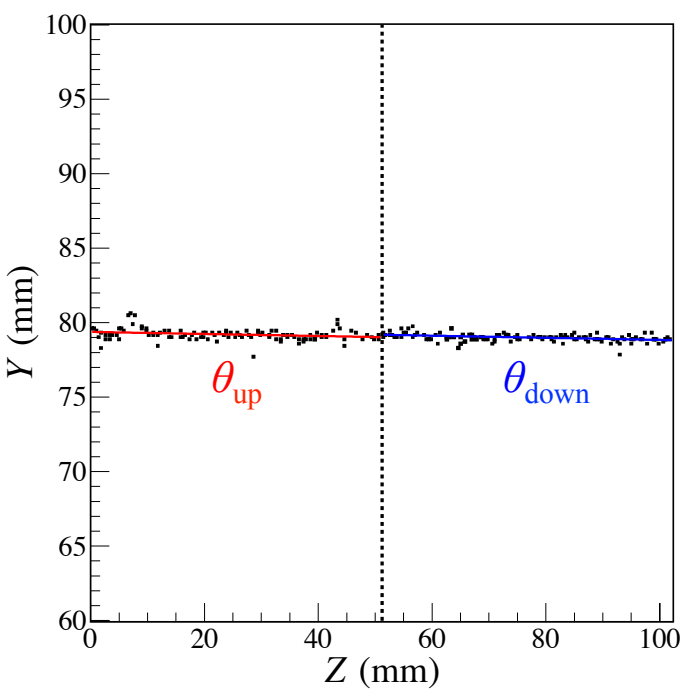

Figure 10: Example of a ${ }^{4} \mathrm{He}$ beam track of the anode strips. The anode strips were perpendicular to the beam axis. The $Z$ coordinate was determined by the strip number multiplied by the strip pitch of $400 \mu \mathrm{m}$, and the $Y$ coordinate was determined by the electron drift time multiplied by the drift velocity. Only the middle clocks between the leading edge and the trailing edge are shown. The track was separated into upstream and downstream regions and each track was fitted to a straight line (solid line) to determine the angle of the beam.

bution was determined by fitting a Gaussian function (the red line in the figure). Assuming the angular resolution for the upstream and the downstream regions to be the same, the angular resolution for the beam particles was400 calculated as $\sigma_{\text {beam }}=\sigma_{\text {up-down }} / \sqrt{2}$.

Figure 12 shows the angular resolution measured at various ${ }^{4} \mathrm{He}$ beam intensities. Whereas operation with the $\mathrm{He}+\mathrm{CO}_{2}$ gas was unstable at beam intensities higher than kcps due to the discharge of the $\mu$-PIC, stable opera-40 tion at beam intensities higher than $1000 \mathrm{kcps}$ was possible with the $\mathrm{He}+$ iso- $\mathrm{C}_{4} \mathrm{H}_{10}$ gas. We note that the high voltage applied to the anode of the $\mu$-PIC was increased to $550 \mathrm{~V}$ during the measurements with beam intensities of 519 and 1061 kcps to keep the pulse height for the beam ${ }_{410}$ particles same as that for the lower beam intensity. The angular resolution worsened with increasing beam intensity because of the buildup of space charge inside the field cage.

\section{Scattering events}

\subsection{Examples of tracks}

Scattering events of the ${ }^{4} \mathrm{He}$ beam off the TPC gas were acquired to develop an event reconstruction algo- ${ }^{42}$ rithm. The TPC was operated with the $\mathrm{He}(93 \%)+$ iso$\mathrm{C}_{4} \mathrm{H}_{10}(7 \%)$ gas at $430 \mathrm{hPa}$. The intensity of the ${ }^{4} \mathrm{He}$ beam was $50 \mathrm{kcps}$. The trigger signals for the scattering events were generated by the Si detectors installed at left and right sides of the TPC.

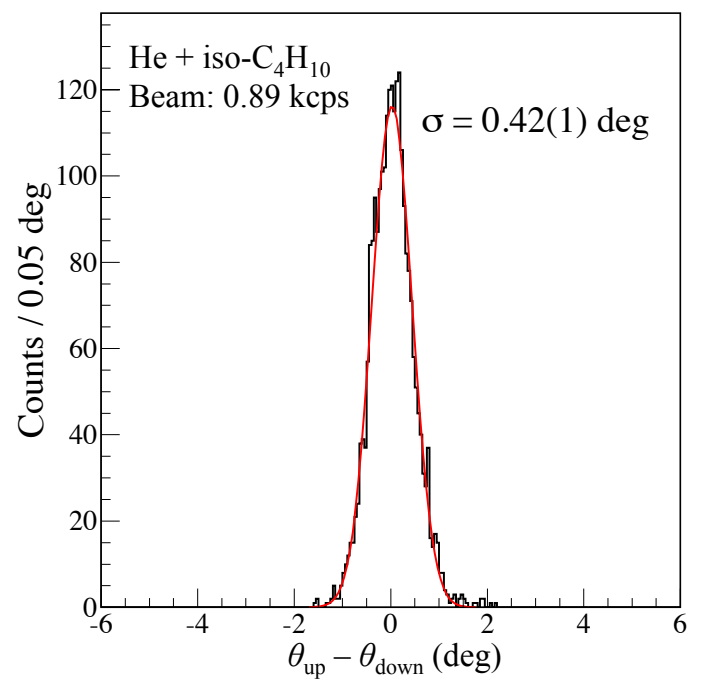

Figure 11: The distribution of the difference of the beam angle between the upstream and the downstream regions. Each angle was calculated by the fitting the tracks to a straight line. The histogram was fitted by a Gaussian function (red solid line) to determine the angular resolution.

The acquired scattering data contains not only the ${ }^{4} \mathrm{He}+{ }^{4} \mathrm{He}$ elastic scattering events, but also scattering from ${ }^{1} \mathrm{H}$ or ${ }^{12} \mathrm{C}$ in the iso- $\mathrm{C}_{4} \mathrm{H}_{10}$ gas. Typical measured tracks are presented in Figs. 13 and 14 . The $X$ and $Z$ coordinates were determined from the cathode and anode strip numbers on the $\mu$-PIC, and the $Y$ coordinate was determined from the electron drift time multiplied by the drift velocity.

Figure 13 shows the tracks of a ${ }^{4} \mathrm{He}+{ }^{4} \mathrm{He}$ elastic scattering event. The incident ${ }^{4} \mathrm{He}$ beam (labeled as ${ }^{4} \mathrm{He}_{3}$ in the figure) was scattered from the ${ }^{4} \mathrm{He}$ gas inside the TPC sensitive volume. From the vertex point, the recoil and the scattered ${ }^{4} \mathrm{He}$ particles (denoted by ${ }^{4} \mathrm{He}_{4}$ and ${ }^{4} \mathrm{He}_{5}$, respectively) were emitted and escaped from the TPC. The tracks of the unreacted pile-up beam particles (denoted as ${ }^{4} \mathrm{He}_{1}$ and ${ }^{4} \mathrm{He}_{2}$, respectively) were also recorded at the same time. The particles ${ }^{4} \mathrm{He}_{1}$ and ${ }^{4} \mathrm{He}_{2}$ did not actually pass at $Y \sim 80 \mathrm{~mm}$ and $Y \sim 140 \mathrm{~mm}$, respectively. The heights of the tracks of these particles were wrongly determined because their arrival timings were different from that of ${ }^{4} \mathrm{He}_{3}$. In addition to the ${ }^{4} \mathrm{He}$ tracks, small points, which do not belong to the particle trajectories, were also recorded. These signals could be attributed to the noise in the circuit or $\mathrm{X}$ rays from the gas atoms.

Figure 14 shows the tracks of a ${ }^{4} \mathrm{He}+{ }^{12} \mathrm{C}$ inelastic scattering event. The ${ }^{4} \mathrm{He}$ beam $\left({ }^{4} \mathrm{He}_{\text {in }}\right)$ was scattered from ${ }^{12} \mathrm{C}$ in the iso- $\mathrm{C}_{4} \mathrm{H}_{10}$ gas and exited the TPC $\left({ }^{4} \mathrm{He}_{\text {out }}\right)$. The ${ }^{12} \mathrm{C}$ target nucleus was excited to above the alpha decay threshold at $E_{x}=7.27 \mathrm{MeV}$, and as a result, three alpha particles $\left(\alpha_{1}, \alpha_{2}\right.$ and $\left.\alpha_{3}\right)$ were emitted. While $\alpha_{1}$ and $\alpha_{2}$ escaped from the TPC and were detected by the Si detectors, $\alpha_{3}$ stopped inside the TPC. Because these decay alpha particles lost more energies than ${ }^{4} \mathrm{He}_{\text {in }}$ and 


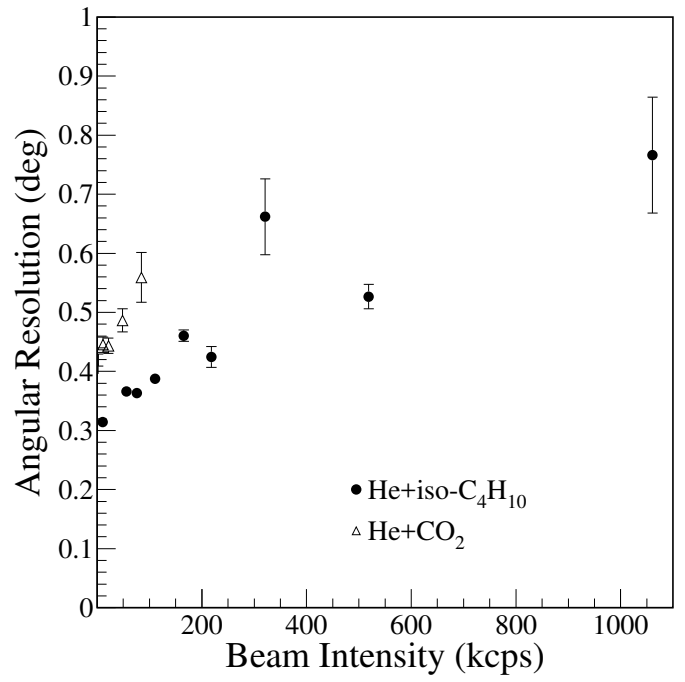

Figure 12: Angular resolution of the TPC for the beam particles $\left({ }^{4} \mathrm{He}\right.$ at $\left.53 \mathrm{MeV}\right)$ as a function of the beam intensity. The solid circles represent the resolution with the $\mathrm{He}(93 \%)+$ iso- $\mathrm{C}_{4} \mathrm{H}_{10}(7 \%)$ gas mixture and the open triangles represent the resolution with the $\mathrm{He}(93 \%)+\mathrm{CO}_{2}(7 \%)$ gas mixture. The pressure of the gasses was ${ }^{475}$ $430 \mathrm{hPa}$. The beam intensities were increased until the discharge occurred at the $\mu$-PIC.

${ }^{4} \mathrm{He}_{\text {out }}$, the induced pulse heights were higher. Thus the ${ }_{480}$ TOTs became longer, and the observed tracks of the decayed particles were thicker.

As seen in Figs. 13 and 14 many trajectories of incident particles, scattered particles, decay particles, unreacted particles, etc. were recorded in the scattering events.485 For the reconstruction of the scattering events, each trajectory in the anode and cathode images must be extracted separately. Here, we adopted an image processing algorithm, namely the Hough transform method.

\subsection{Track finding algorithm}

The Hough transform method was originally invented to analyze the bubble chamber pictures [46. It allows the extraction of features such as lines and circles in a picture without knowing how many features are contained. 495 This algorithm is now widely applied to image recognition because of its high immunity to random noise in images compared to other methods like the least-square technique.

The anode and cathode track images obtained by MAIKo contain 256-strip $\times$ 1024-clock pixels. The coordinates of ${ }_{500}$ the $i$-th pixel are expressed as $\left(x_{i}, y_{i}\right)$ in the track space, as illustrated in the left panel of Fig. 15. In the Hough transformation, a hit pixel at $\left(x_{i}, y_{i}\right)$ is transformed into a curved line in the $(\theta, r)$ parameter space (Hough space) according to the following formula [47]:

$$
r=x_{i} \cos \theta+y_{i} \sin \theta .
$$

For example, the points at $\left(x_{1}, y_{1}\right),\left(x_{2}, y_{2}\right)$, and $\left(x_{3}, y_{3}\right)$ in the track space are transformed into the red-solid, green- one straight line in the track space as given by the following formula.

$$
y=-\frac{x}{\tan \theta_{j}}+\frac{r_{j}}{\sin \theta_{j}} .
$$

${ }_{460}$ As shown in the left panel of Fig. 15, $r_{j}$ corresponds to the distance between the straight line and the origin. $\theta_{j}$ corresponds to the angle between the $x$-axis and the perpendicular line from the origin.

If the pixels in the anode or cathode track space lie on 465 a straight line, their transformed curves intersect at one point at $\left(\theta_{j}, r_{j}\right)$ in the Hough space. Thus, the coordinates of the intersection point give the particle track according to Eq. (2).

In the present analysis, the transformed curves were discretized into pixels and these pixels were booked into a two-dimensional histogram in the Hough space. For example, the anode image in Fig. 13(a) was transformed into the Hough histogram shown in Fig. 16. The bin size of the histogram was set to $2^{\circ} \times 0.78 \mathrm{~mm}$. The tracks with sizable length and width made clear peaks as labeled and circled in the Hough histogram, whereas the small spots caused by noise did not. By finding the pixel with the maximum count in the Hough histogram, which is easy to implement in a computer program, the longest track in the anode image was determined.

After the first track was extracted, pixels along the first track were eliminated from the image, and the remaining pixels were again transformed to the Hough space. The maximum pixel in the second Hough histogram provided the second track in the image. The above procedures were repeated until the number of content in the maximum pixel became lower than a threshold (150 counts). In this way, multiple tracks were separately determined.

The tracks extracted from the anode image in Fig. 13(a) are shown as the red-solid lines in the figures. In the cathode track finding, the image was divided into two regions: $X<40 \mathrm{~mm}$ and $X>60 \mathrm{~mm}$ in order to avoid the beam tracks because the beam tracks were observed as the circular spots around $X=50 \mathrm{~mm}$ in the cathode image as seen in Fig. 13(b) The cathode strips at $40 \mathrm{~mm}<X<60 \mathrm{~mm}$ were not taken into account in the track finding. The tracks in the $X<40 \mathrm{~mm}$ and $X>60$ $\mathrm{mm}$ regions were independently extracted as indicated by the green-dashed line and the magenta-dotted lines, respectively. The track of $\alpha_{3}$ was not extracted from the cathode image because this track remained within $40 \mathrm{~mm}$ $<X<60 \mathrm{~mm}$.

\subsection{Analysis of the ${ }^{4} \mathrm{He}+{ }^{4} \mathrm{He}$ elastic scattering}

Because the relative angle between the scattered and the recoil particles is always $90^{\circ}$ in the elastic scattering of identical particles, this reaction is a suitable benchmark to evaluate the angular resolution of the detector.

After the track finding in the anode and cathode images by the Hough transform algorithm was finished, the 


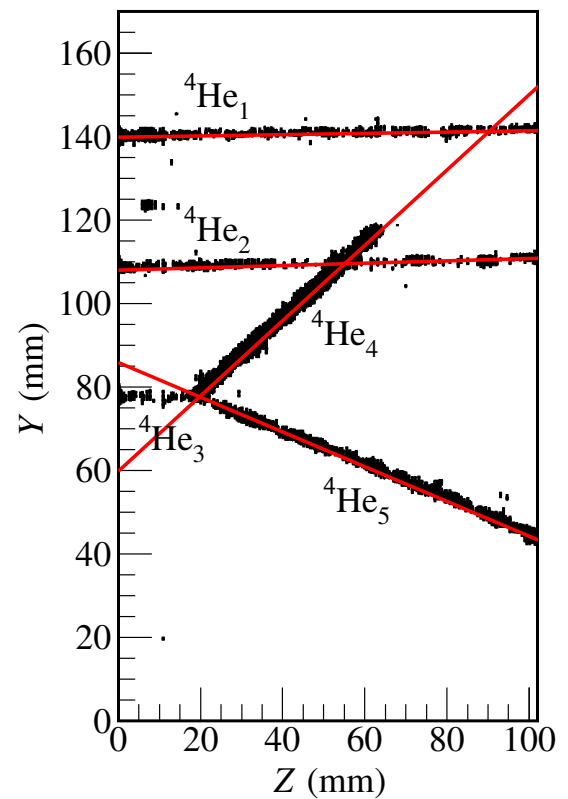

(a) Anode track.

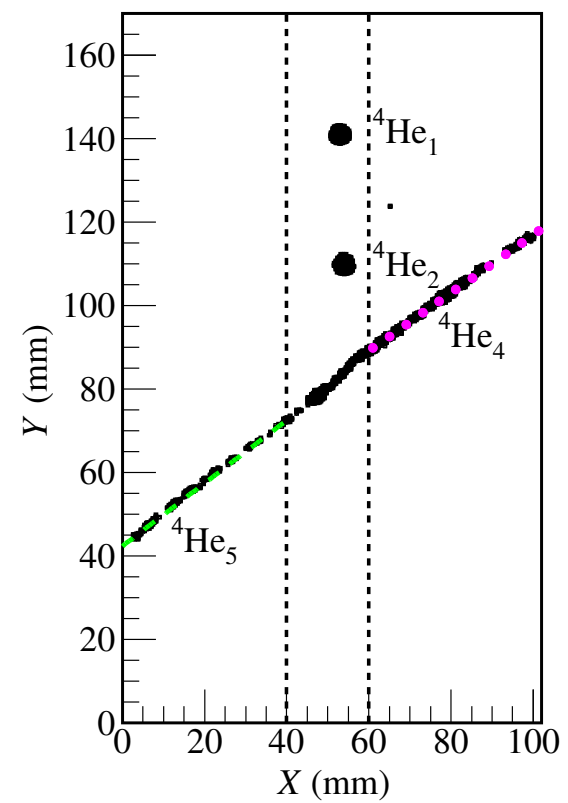

(b) Cathode track.

Figure 13: Track sample of a ${ }^{4} \mathrm{He}+{ }^{4} \mathrm{He}$ scattering. The $Z$ position in the left panel and the $X$ position in the right panel were determined from the $\mu$-PIC strip number. The $Y$ position in both panels were determined from the electron drift time multiplied by the electron drift velocity. The red-solid lines, green-dashed line, and magenta-dotted line represent the tracks identified by the Hough transform algorithm described in the text. The vertical dashed lines are drawn at $X=40$ and $60 \mathrm{~mm}$.

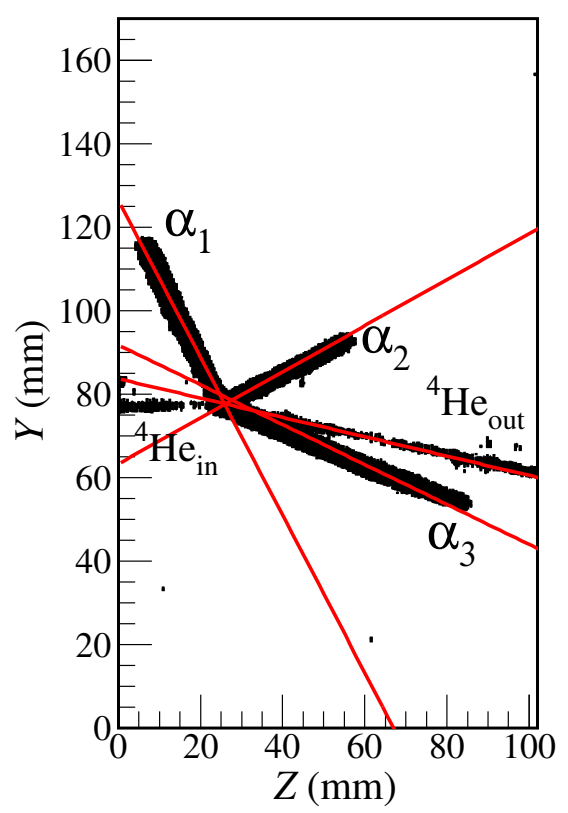

(a) Anode track

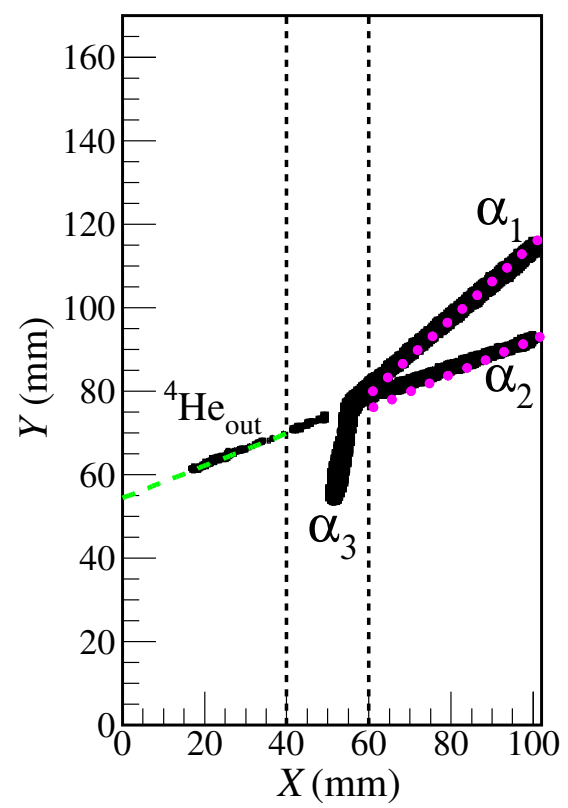

(b) Cathode track

Figure 14: Similar to Fig. 13 but for a ${ }^{4} \mathrm{He}+{ }^{12} \mathrm{C}$ inelastic scattering. The ${ }^{12} \mathrm{C}$ nucleus contained in the iso- $\mathrm{C}_{4} \mathrm{H}_{10}$ gas decayed into three alpha particles $\left(\alpha_{1}, \alpha_{2}\right.$, and $\left.\alpha_{3}\right)$ immediately after being excited to above the alpha decay threshold. 

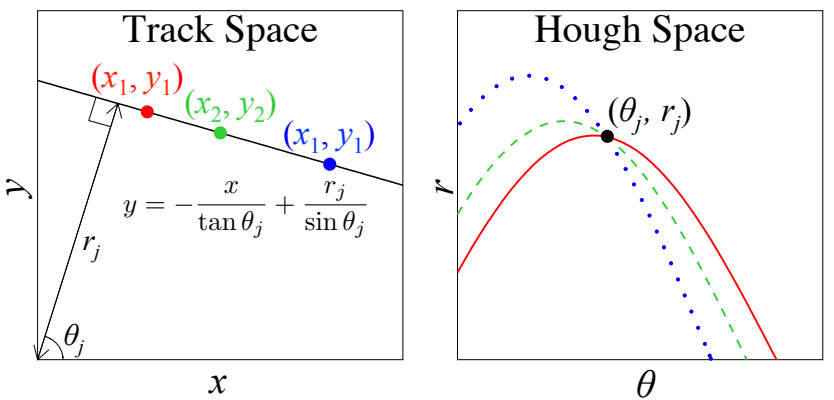

Figure 15: Example of the Hough transformation. Three points ${ }^{525}$ at $\left(x_{1}, y_{1}\right),\left(x_{2}, y_{2}\right)$, and $\left(x_{3}, y_{3}\right)$ in the left panel are transformed into the red-solid, green-dashed, and blue-dotted curves, respectively, according to Eq. (1). The intersection point of the curves $\left(\theta_{j}, r_{j}\right)$ gives the equation of the line in the track space.

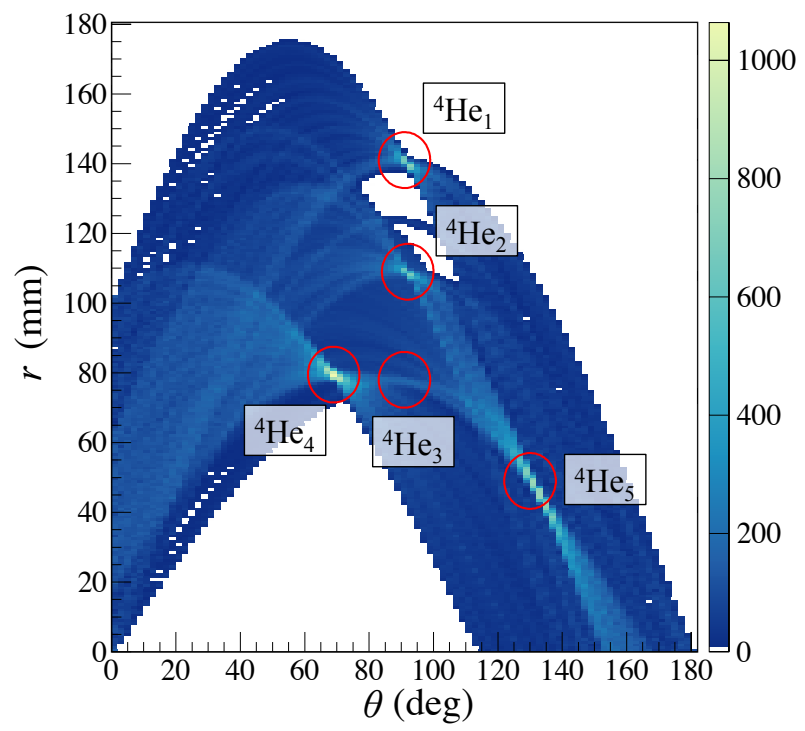

Figure 16: Two-dimensional histogram in the Hough space made from the anode image shown in Fig $13(\mathrm{a})$ The circled peaks correspond to the particle trajectories in Fig. 13(a) following two conditions were imposed to identify candidates for elastic scattering events. First, two tracks with tilted angles more than $4^{\circ}$ from the $Z$-axis were found in the anode image. Second, a single track was found in both of the $X<40 \mathrm{~mm}$ and $X<60 \mathrm{~mm}$ regions of the cathode image. The tracks in Fig. 13 satisfy the above conditions, whereas the tracks in Fig. 14 do not because three tilted tracks were observed in the anode image and the two tracks were observed in the $X>60 \mathrm{~mm}$ region of the cathode image (magenta-dotted lines).

The three dimensional trajectories of the two particles observed in the candidates for the elastic scattering events were reconstructed by combining the two tracks in the anode and cathode images, and the opening angle between the two particles was determined. The reconstructed opening angle between the two trajectories $\left(\theta_{3}+\theta_{4}\right)$ is shown in Fig. 17. The black line shows the histogram of the opening angle calculated directly from the discretized $\theta$ values of the peaks in the Hough histograms. The distribution shows a clear peak at $90^{\circ}$, and this result suggests that the elastic scattering events were successfully identified in the present analysis.

The standard deviation of the distribution $\left[\sigma\left(\theta_{3}+\theta_{4}\right)\right]$ is $3.9^{\circ}$. This angular resolution can be improved by further analysis, because the accuracy of the present analysis is limited by the granularity of the Hough histogram. In the present analysis, the angular bin size of the Hough histogram was $2^{\circ}$. With smaller bin size, we can expect to improve the angular resolution. However, the analysis with a finer segmented Hough histogram will be computationally expensive. In addition, the number of the contents of each bin will decrease, which will reduce the contrast of the histogram and make it difficult to determine the peak in the Hough histogram.

Instead of such an expensive analysis, the discretized $\theta_{j}$ and $r_{j}$ values were corrected by fitting Eq. (2) to the hit pixels in the track space. For the correction, only the middle points between the leading edges and the trailing edges in the anode and cathode images were included in the fit. The red hatched histogram in Fig. 17 shows the histogram of the opening angle between the two trajectories calculated from the corrected $\theta$ values. The standard deviation becomes $1.9^{\circ}$, which is about two times better than the one before the correction. Assuming that the angular resolutions for two trajectories are the same, the angular resolution for one trajectory is calculated to be $\sigma=\sigma\left(\theta_{3}+\theta_{4}\right) / \sqrt{2}=1.3^{\circ}$. This resolution agrees with the result from a Monte-Carlo simulation assuming the in-plane angular resolution of $0.4^{\circ}$ discussed in Section 4.2 and taking into account the error propagation in the three-dimensional track reconstruction from the anode and cathode images.

Angular resolution achieved by the existing TPC based active targets is around $1^{\circ}$ [48, 49]. MAIKo demonstrates comparable performance to these active targets. 


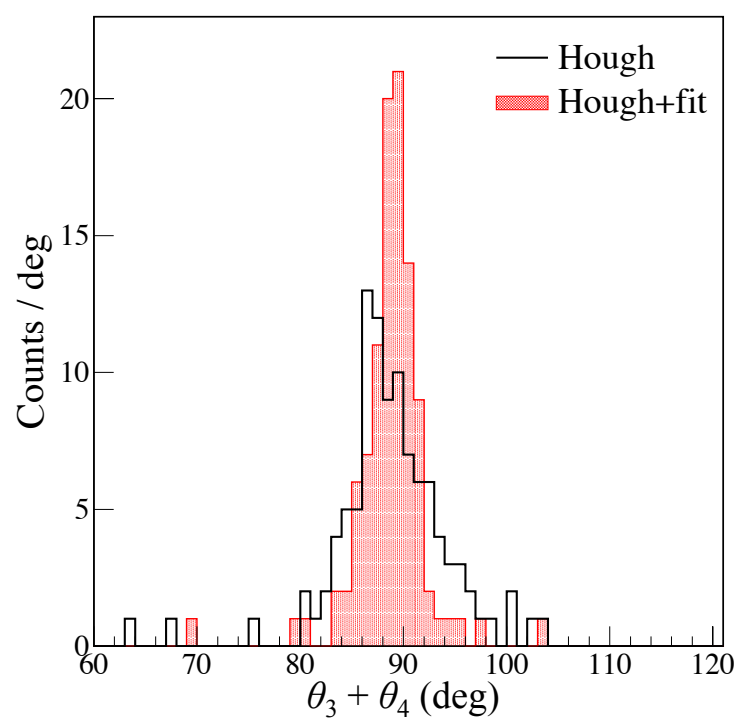

Figure 17: Distribution of the relative angle between the two emitted particles in the ${ }^{4} \mathrm{He}+{ }^{4} \mathrm{He}$ elastic scattering. The black open histogram represents the angle determined directly from the Hough transformation $\left[\sigma\left(\theta_{3}+\theta_{4}\right)=3.9^{\circ}\right]$. The angle obtained by the fitting after the Hough transformation is shown as the red hatched histogram $\left[\sigma\left(\theta_{3}+\theta_{4}\right)=1.9^{\circ}\right]$.

\section{Summary and outlook}

An active target system MAIKo has been developed ${ }_{620}$ by Kyoto University and RCNP for use in missing-mass spectroscopy on unstable nuclei in inverse kinematics. By detecting the recoil particles inside the detector volume, measurements at forward angles are enabled.

The TPC was commissioned with the $\mathrm{He}+$ iso- $\mathrm{C}_{4} \mathrm{H}_{10}$ and $\mathrm{He}+\mathrm{CO}_{2}$ gas mixtures at $430 \mathrm{hPa}$. An electric field of about $200 \mathrm{~V} / \mathrm{cm}$ was applied where the electron drift velocity was about $1.5 \mathrm{~cm} / \mu \mathrm{s}$. The maximum gas gain 630 achieved by the $\mu$-PIC was about 3000 .

The detector performances were examined by using a $56-\mathrm{MeV}{ }^{4} \mathrm{He}$ beam at RCNP. The angular resolution of the TPC for the beam particle was measured under various635 beam intensities. The TPC was successfully operated with the $\mathrm{He}+\mathrm{iso}-\mathrm{C}_{4} \mathrm{H}_{10}$ gas even when the beam intensity was higher than 1000 kcps. However, the operation was not stable at $100 \mathrm{kcps}$ due to the discharge of the $\mu$-PIC when640 the $\mathrm{He}+\mathrm{CO}_{2}$ gas was used.

Events in which the beam particles were scattered from the TPC gas were acquired. A track finding algorithm based on the Hough transformation was developed. The645 tracking algorithm was benchmarked by analyzing the ${ }^{4} \mathrm{He}$ $+{ }^{4} \mathrm{He}$ elastic scattering events. Multiple tracks were successfully separated into the individual tracks. The angular resolution for the scattered particles was successfully im-650 proved to $1.3^{\circ}$ in sigma by applying the fitting of the track after the Hough transformation; the result is consistent with the in-plane angular resolution of $0.4^{\circ}$ determined for beam particles.
Recently, the first measurement of the alpha inelastic scattering using an RI beam has been completed at RCNP. A ${ }^{10} \mathrm{C}$ secondary beam with an energy of $75 \mathrm{MeV} /$ nucleon was injected into MAIKo. The intensity of the beam was about $80 \mathrm{kcps}$. According to the online analysis, the detecthreshold for the recoil alpha particles was less than $500 \mathrm{keV}$. The detailed analysis is on going and the results will be reported elsewhere in the near future.

\section{Acknowledgements}

The authors are grateful to the cyclotron crews at RCNP 605 for providing the high-quality and stable ${ }^{4} \mathrm{He}$ beam. The authors also thank Dr. H. Baba from RIKEN for his valuable advice on the data acquisition system. T. F. appreciates the JSPS Research Fellowship for young scientists under program No. JP14J00949. H. J. O and I. T. acknowledge the support of A. Tohsaki and his spouse. The present work was supported by JSPS KAKENHI Grant Nos. JP20244030, JP23340068, JP23224008 and 15H02091.

\section{References}

[1] G. R. Satchler, Direct Nuclear Reactions, Clarendon Press, 1983.

[2] Y. Yano, The RIKEN RI Beam Factory Project: A status report, Nuclear Instrument and Methods in Physics Research Section B 261 (2007) 1009 - 1013. doi:10.1016/j.nimb.2007.04. 174

[3] M. Thoennessen, Plans for the Facility for Rare Isotope Beams, Nuclear Physics Section A 834 (2010) 688c - 693c. doi:10. 1016/j.nuclphysa.2010.01.125

[4] P. Spiller, G. Franchetti, The FAIR accelerator project at GSI, Nuclear Instrument and Methods in Physics Research Section A 561 (2006) 305 - 309. doi:10.1016/j.nima.2006.01.043

[5] S. Gales, SPIRAL2 at GANIL: Next Generation of ISOL Facility for Intense Secondary Radioactive Ion Beams, Nuclear Physics

(1) A 834 (2010) 717c - 723c. doi:10.1016/j.nuclphysa.2010.01. 130

[6] H. Moeini, S. Ilieva, F. Aksouh, K. Boretzky, A. Chatillon, A. Corsi, P. Egelhof, H. Emling, G. Ickert, J. Jourdan, N. K. Nayestanaki, D. Kiselev, O. Kiselev, C. Kozhuharov, T. L. Bleis, X. Le, Y. Litvinov, K. Mahata, J. Meier, F. Nolden, S. Paschalis, U. Popp, H. Simon, M. Steck, T. Stöhlker, H. Weick, D. Werthmüller, A. Zalite, First feasibility experiment for the EXL project with prototype detectors at the ESR storage ring, Nuclear Instrument and Methods in Physics Research Section A 634 (2011) 77 - 84. doi:10.1016/j.nima.2011.01.036

[7] M. von. Schmid, S. Bagchi, S. Bönig, M. Csatlós, I. Dillmann, C. Dimopoulou, P. Egelhof, V. Eremin, T. Furuno, H. Geissel, R. Gernhäuser, M. N. Harakeh, A.-L. Hartig, S. Illieva, N. Kalantar-Nayestanaki, O. Kiselev, H. Kollmus, C. Kozhuharov, A. Krasznahorkay, T. Kröll, M. Kuilman, S. Litvinov, Y. A. Litvinov, M. Mahjour-Shafiei, M. Mutterer, D. Nagae, M. A. Najafi, C. Nociforo, F. Nolden, U. Popp, C. Rigollet, S. Roy, C. Scheidenberger, M. Steck, B. Streicher, L. Stuhl, M. Thürauf, T. Uesaka, H. Weick, J. S. Winfield, D. Winters, P. J. Woods, T. Yamaguchi, K. Yue, J. C. Zamora, J. Zenihiro, First EXL experiment with stored radioactive beam: Proton scattering on ${ }^{56} \mathrm{Ni}$, EPJ Web of Conferences 66 (2014) 03093. doi:10.1051/epjconf/20146603093

[8] J. C. Zamora, T. Aumann, S. Bagchi, S. Bönig, M. Csatlós, I. Dillmann, C. Dimopoulou, P. Egelhof, V. Eremin, T. Furuno, H. Geissel, R. Gernhäuser, M. N. Harakeh, A.-L. Hartig, S. Ilieva, N. Kalantar-Nayestanaki, O. Kiselev, H. Kollmus, C. Kozhuharov, A. Krasznahorkay, T. Kröll, M. Kuilman, 
S. Litvinov, Y. A. Litvinov, M. Mahjour-Shafiei, M. Mutterer, D. Nagae, M. Najafi, C. Nociforo, F. Nolden, U. Popp, C. Rigollet, S. Roy, C. Scheidenberger, M. von. Schmid, M. Steck,730 B. Streicher, L. Stuhl, M. Thürauf, T. Uesaka, H. Weick, J. S. Winfield, D. Winters, P. J. Woods, T. Yamaguchi, K. Yue, J. Zenihiro, First measurement of isoscalar giant resonances in a stored-beam experiment, Physics Letters B 763 (2016) 16 19. doi:10.1016/j.physletb.2016.10.015

9] J. C. Zamora, T. Aumann, S. Bagchi, S. Bönig, M. Csatlós, I. Dillmann, C. Dimopoulou, P. Egelhof, V. Eremin, T. Furuno, H. Geissel, R. Gernhäuser, M. N. Harakeh, A.-L. Hartig, S. Ilieva, N. Kalantar-Nayestanaki, O. Kiselev, H. Kollmus, C. Kozhuharov, A. Krasznahorkay, T. Kröll, M. Kuilman,740 S. Litvinov, Y. A. Litvinov, M. Mahjour-Shafiei, M. Mutterer, D. Nagae, M. Najafi, C. Nociforo, F. Nolden, U. Popp, C. Rigollet, S. Roy, C. Scheidenberger, M. von. Schmid, M. Steck, B. Streicher, L. Stuhl, M. Thürauf, T. Uesaka, H. Weick, J. S. Winfield, D. Winters, P. J. Woods, T. Yamaguchi, K. Yue,745 J. Zenihiro, Nuclear-matter radius studies from ${ }^{58} \mathrm{Ni}(\alpha, \alpha)$ experiments at the GSI Experimental Storage Ring with the EXL facility, Physical Review C 96 (2017) 034617. doi:10.1103/ PhysRevC.96.034617

[10] S. Neumaier, G. Alkhazov, M. Andronenko, A. Dobrovol-750 sky, P. Egelhof, G. Gavrilov, H. Geissel, H. Irnich, A. Khanzadeev, G. Korolev, A. Lobodenko, G. Münzenberg, M. Mutterer, W. Schwab, D. Seliverstov, T. Suzuki, N. Timofeev, A. Vorobyov, V. Yatsoura, Small-angle proton elastic scattering from the neutron-rich isotopes ${ }^{6} \mathrm{He}$ and ${ }^{8} \mathrm{He}$, and from ${ }^{4} \mathrm{He}$, at755 $0.7 \mathrm{GeV}$ in inverse kinematics, Nuclear Physics A 712 (2002) 247 - 268. doi:10.1016/S0375-9474(02)01274-5

[11] T. Hashimoto, H. Ishiyama, T. Ishikawa, T. Kawamura, K. Nakai, Y. Watanabe, H. Miyatake, M. Tanaka, Y. Fuchi, N. Yoshikawa, S. Jeong, I. Katayama, T. Nomura, T. Furukawa,760 S. Mitsuoka, K. Nishio, M. Matsuda, H. Ikezoe, T. Fukuda, S. Das, P. Saha, Y. Mizoi, T. Komatsubara, M. Yamaguchi, Y. Tagishi, Gated multiple-sampling and tracking proportional chamber: New detector system for nuclear astrophysical study with radioactive nuclear beams, Nuclear Instrument and Meth-765 ods in Physics Research Section A 556 (2006) 339 - 349. doi:10.1016/j.nima.2005.10.018

[12] C. Demonchy, M. Caaman̄o, H. Wang, W. Mittig, P. RousselChomaz, H. Savajols, M. Chartier, D. Cortina-Gil, A. Fomichev, G. Frémont, P. Gangnant, A. Gillibert, L. Giot, M. Golovkov,770 B. Jurado, J. Libin, A. Obertelli, E. Pollaco, A. Rodin, C. Spitaels, S. Stepantsov, G. Ter-Akopian, R. Wolski, MAYA: An active-target detector for binary reactions with exotic beams, Nuclear Instrument and Methods in Physics Research Section A 583 (2007) 341 - 349. doi:10.1016/j.nima.2007.09.022 77

[13] D. Suzuki, M. Ford, D. Bazin, W. Mittig, W. G. Lynch, T. Ahn, S. Aune, E. Galyaev, A. Fritsch, J. Gilbert, F. Montes, A. Shore, J. Kolata, J. Browne, A. Howard, A. Roberts, X. Tang, Prototype AT-TPC: Toward a new generation active target time projection chamber for radioactive beam experiments, Nuclear780 Instrument and Methods in Physics Research Section A 691 (2012) 39 - 54. doi:10.1016/j.nima.2012.06.050

[14] S. Ota, H. Tokieda, C. S. Lee, Y. N. Watanabe, CNS active target (CAT) for missing mass spectroscopy with intense beams, Journal of Radioanalytical and Nuclear Chemistry 305 (2015) 785 907 - 911. doi:10.1007/s10967-015-4130-5

[15] J. Bradt, D. Bazin, F. Abu-Nimeh, T. Ahn, Y. Ayyad, S. B. Novo, L. Carpenter, M. Cortesi, M. Kuchera, W. Lynch, W. Mittig, S. Rost, N. Watwood, J. Yurkon, Commissioning of the Active-Target Time Projection Chamber, Nuclear Instru-790 ment and Methods in Physics Research Section A 875 (2017) 65 - 79. doi:10.1016/j.nima.2017.09.013

[16] T. Roger, J. Pancin, G. Grinyer, B. Mauss, A. Laffoley, P. Rosier, H. Alvarez-Pol, M. Babo, B. Blank, M. Caaman̄o, S. Ceruti, J. Daemen, S. Damoy, B. Duclos, B. Fernández-795 Domínguez, F. Flavigny, J. Giovinazzo, T. Goigoux, J. Henares, P. Konczykowski, T. Marchi, G. Lebertre, N. Lecesne, L. Legeard, C. Maugeais, G. Minier, B. Osmond, J. Pedroza, J. Piber- nat, O. Poleshchuk, E. Pollacco, R. Raabe, B. Raine, F. Renzi, F. Saillant, P. Sénécal, P. Sizun, D. Suzuki, J. Swartz, C. Wouters, G. Wittwer, J. Yang, Demonstrator detection system for the active target and time projection chamber (actar tpc) project, Nuclear Instruments and Methods in Physics Research Section A 895 (2018) 126 - 134. doi:10.1016/j.nima. 2018.04 .003

[17] I. Tanihata, M. Alcorta, D. Bandyopadhyay, R. Bieri, L. Buchmann, B. Davids, N. Galinski, D. Howell, W. Mills, S. Mythili, R. Openshaw, E. Padilla-Rodal, G. Ruprecht, G. Sheffer, A. C. Shotter, M. Trinczek, P. Walden, H. Savajpls, T. Roger, M. Caaman̄o, W. Mittig, P. Roussel-Chomaz, R. Kanungo, A. Gallant, M. Notani, G. Savard, I. J. Thompson, Measurement of the Two-Halo Neutron Transfer Reaction ${ }^{1} \mathrm{H}\left({ }^{11} \mathrm{Li},{ }^{9} \mathrm{Li}\right){ }^{3} \mathrm{H}$ at $3 A \mathrm{MeV}$, Physical Review Letters 100 (2008) 192502. doi:10.1103/PhysRevLett.100.192502

[18] M. Vandebrouck, J. Gibelin, E. Khan, N. L. Achouri, H. Baba, D. Beaumel, Y. Blumenfeld, M. Caaman̄o, L. Càceres, G. Colò, F. Delaunay, B. Fernandez-Dominguez, U. Garg, G. F. Grinyer, M. N. Harakeh, N. Kalantar-Nayestanaki, N. Keeley, W. Mittig, J. Pancin, R. Raabe, T. Roger, P. Roussel-Chomaz, H. Savajols, O. Sorlin, C. Stodel, D. Suzuki, J. C. Thomas, Measurement of the isoscalar monopole response in the neutron-rich nucleus ${ }^{68} \mathrm{Ni}$, Physical Review Letters 113 (2014) 032504. doi:10.1103/ PhysRevLett.113.032504.

[19] M. Vandebrouck, J. Gibelin, E. Khan, N. L. Achouri, H. Baba, D. Beaumel, Y. Blumenfeld, M. Caaman̄o, L. Càceres, G. Colò, F. Delaunay, B. Fernandez-Dominguez, U. Garg, G. F. Grinyer, M. N. Harakeh, N. Kalantar-Nayestanaki, N. Keeley, W. Mittig, J. Pancin, R. Raabe, T. Roger, P. Roussel-Chomaz, H. Savajols, O. Sorlin, C. Stodel, D. Suzuki, J. C. Thomas, Isoscalar response of ${ }^{68} \mathrm{Ni}$ to $\alpha$-particle and deuteron probes, Physical Review C 92 (2015) 024316. doi:10.1103/PhysRevC.92.024316

[20] S. Bagchi, J. Gibelin, M. Harakeh, N. Kalantar-Nayestanaki, N. Achouri, H. Akimune, B. Bastin, K. Boretzky, H. Bouzomita, M. Caamaāo, L. Càceres, S. Damoy, F. Delaunay, B. FernándezDomínguez, M. Fujiwara, U. Garg, G. Grinyer, O. Kamalou, E. Khan, A. Krasznahorkay, G. Lhoutellier, J. Libin, S. Lukyanov, K. Mazurek, M. Najafi, J. Pancin, Y. Penionzhkevich, L. Perrot, R. Raabe, C. Rigollet, T. Roger, S. Sambi, H. Savajols, M. Senoville, C. Stodel, L. Suen, J. Thomas, M. Vandebrouck, J. V. de Walle, Observation of isoscalar multipole strengths in exotic doubly-magic ${ }^{56} \mathrm{Ni}$ in inelastic $\alpha$ scattering in inverse kinematics, Physics Letters B 751 (2015) 371 - 375. doi:10.1016/j.physletb.2015.10.060

[21] C. Rodríguez-Tajes, F. Farget, L. Acosta, H. Alvarez-Pol, M. Babo, F. Boulay, M. Caaman̄o, S. Damoy, B. FernándezDomínguez, D. Galaviz, G. Grinyer, J. Grinyer, M. Harakeh, P. Konczykowski, I. Martel, J. Pancin, G. Randisi, F. Renzi, T. Roger, A. Sánchez-Benítez, P. Teubig, M. Vandebrouck, First inverse-kinematics fission measurements in a gaseous active target, Nuclear Physics A 958 (2017) 246 - 265. doi: 10.1016/j.nuclphysa.2016.12.003

[22] D. Suzuki, A. Shore, W. Mittig, J. J. Kolata, D. Bazin, M. Ford, T. Ahn, F. D. Becchetti, S. Beceiro Novo, D. Ben Ali, B. Bucher, J. Browne, X. Fang, M. Febbraro, A. Fritsch, E. Galyaev, A. M. Howard, N. Keeley, W. G. Lynch, M. Ojaruega, A. L. Roberts, X. D. Tang, Resonant $\alpha$ scattering of ${ }^{6} \mathrm{He}$ : Limits of clustering in ${ }^{10} \mathrm{Be}$, Physical Review C 87 (2013) 054301. doi:10.1103/ PhysRevC.87.054301

[23] A. Fritsch, S. Beceiro-Novo, D. Suzuki, W. Mittig, J. J. Kolata, T. Ahn, D. Bazin, F. D. Becchetti, B. Bucher, Z. Chajecki, X. Fang, M. Febbraro, A. M. Howard, Y. Kanada-En'yo, W. G. Lynch, A. J. Mitchell, M. Ojaruega, A. M. Rogers, A. Shore, T. Suhara, X. D. Tang, R. Torres-Isea, H. Wang, One-dimensionality in atomic nuclei: A candidate for linearchain $\alpha$ clustering in ${ }^{14} \mathrm{C}$, Physical Review C 93 (2016) 014321. doi:10.1103/PhysRevC.93.014321

[24] J. Bradt, Y. Ayyad, D. Bazin, W. Mittig, T. Ahn, S. B. Novo, B. Brown, L. Carpenter, M. Cortesi, M. Kuchera, W. Lynch, S. Rost, N. Watwood, J. Yurkon, J. Barney, U. Datta, J. Es- 
tee, A. Gillibert, J. Manfredi, P. Morfouace, D. Pérez-Loureiro,870 E. Pollacco, J. Sammut, S. Sweany, Study of spectroscopic factors at $N=29$ using isobaric analogue resonances in inverse kinematics, Physics Letters B 778 (2018) 155 - 160. doi:10.1016/j.physletb.2018.01.015

[25] S. Beceiro-Novo, T. Ahn, D. Bazin, W. Mittig, Active targets875 for the study of nuclei far from stability, Progress in Particle and Nuclear Physics 84 (2015) 124 - 165. doi:10.1016/j.ppnp. 2015.06 .003

[26] T. Shimoda, H. Miyatake, S. Morinobu, Design study of the secondary-beam line at RCNP, Nuclear Instrument and Meth-880 ods in Physics Research Section B 70 (1992) 320 - 330. doi: 10.1016/0168-583X (92) 95948-Q

[27] S. Mitsuoka, T. Shimoda, H. Miyatake, Y. Mizoi, H. Kobayashi, M. Sasaki, T. Shirakura, N. Takahashi, T. Murakami, S. Morinobu, Performance of fragment separator with homogeneously885 thick energy degrader: a highly flexible system for use with degraders in a wide thickness range, Nuclear Instrument and Methods in Physics Research Section A 372 (1996) 489 - 500. doi:10.1016/0168-9002(95)01299-0

[28] A. Ochi, T. Nagayoshi, S. Koishi, T. Tanimori, T. Nagae,890 M. Nakamura, A new design of the gaseous imaging detector: Micro pixel chamber, Nuclear Instrument and Methods in Physics Research Section A 471 (2001) 264 - 267. doi: 10.1016/S0168-9002(01) 00996-2

[29] Y. Mizumura, T. Tanimori, H. Kubo, A. Takada, J. D. Parker,895 T. Mizumoto, S. Sonoda, D. Tomono, T. Sawano, K. Nakamura, Y. Matsuoka, S. Komura, S. Nakamura, M. Oda, K. Miuchi, S. Kabuki, Y. Kishimoto, S. Kurosawa, S. Iwaki, Development of a $30 \mathrm{~cm}$-cube Electron-Tracking Compton Camera for the SMILE-II Experiment, Journal of Instrumentation 9 (2014)900 C05045. doi: 10.1088/1748-0221/9/05/C05045

[30] D. Tomono, T. Mizumoto, A. Takada, S. Komura, Y. Matsuoka, Y. Mizumura, M. Oda, T. Tanimori, Establishment of Imaging Spectroscopy of Nuclear Gamma-Rays based on

n Geometrical Optics, Scientific Reports 7 (2017) 41972. doi:005 $10.1038 /$ srep41972

[31] T. Tanimori, Y. Mizumura, A. Takada, S. Miyamoto, T. Takemura, T. Kishimoto, S. Komura, H. Kubo, S. Kurosawa, Y. Matsuoka, K. Miuchi, T. Mizumoto, Y. Nakamasu, K. Nakamura, J. D. Parker, T. Sawano, S. Sonoda, D. Tomono,910 K. Yoshikawa, First On-Site True Gamma-Ray ImagingSpectroscopy of Contamination near Fukushima Plant, Scientific Reports 7 (2017) 41511. doi:10.1038/srep41511

[32] S. Komura, A. Takada, Y. Mizumura, S. Miyamoto, T. Takemura, T. Kishimoto, H. Kubo, S. Kurosawa, Y. Matsuoka,915 K. Miuchi, T. Mizumoto, Y. Nakamatsu, K. Nakamura, M. Oda, J. D. Parker, T. Sawano, S. Sonoda, T. Tanimori, D. Tomono, K. Yoshikawa, Imaging Polarimeter for a Sub-MeV GammaRay All-sky Survey Using an Electron-tracking Compton Camera, The Astrophysical Journal 839 (2017) 41. doi:10.3847/,20 1538-4357/aa68dc

[33] K. Miuchi, H. Nishimura, K. Hattori, N. Higashi, C. Ida, S. Iwaki, S. Kabuki, H. Kubo, S. Kurosawa, K. Nakamura, J. Parker, T. Sawano, M. Takahashi, T. Tanimori, K. Taniue, K. Ueno, H. Sekiya, A. Takeda, K. Tsuchiya, A. Takada, First925 underground results with NEWAGE-0.3a direction-sensitive dark matter detector, Physics Letters B 686 (2010) 11 - 17. doi: $10.1016 / j \cdot$ physletb.2010.02.028

[34] K. Nakamura, K. Miuchi, T. Tanimori, H. Kubo, A. Takada, J. D. Parker, T. Mizumoto, Y. Mizumura, H. Nishimura, H. Sekiya, A. Takeda, T. Sawano, Y. Matsuoka, S. Komura, Y. Yamaguchi, T. Hashimoto, Direction-sensitive dark matter search with gaseous tracking detector NEWAGE-0.3b', Progress of Theoretical and Experimental Physics 2015 (2015) 043F01. doi: $10.1093 / \mathrm{ptep} / \mathrm{ptv041}$

[35] J. Parker, K. Hattori, H. Fujioka, M. Harada, S. Iwaki, S. Kabuki, Y. Kishimoto, H. Kubo, S. Kurosawa, K. Miuchi, T. Nagae, H. Nishimura, T. Oku, T. Sawano, T. Shinohara, J. Suzuki, A. Takada, T. Tanimori, K. Ueno, Neutron imaging detector based on the $\mu$ PIC micro-pixel chamber, Nuclear
Instruments and Methods in Physics Research Section A 697 (2013) 23 - 31. doi:10.1016/j.nima.2012.08.036

[36] J. Parker, M. Harada, K. Hattori, S. Iwaki, S. Kabuki, Y. Kishimoto, H. Kubo, S. Kurosawa, Y. Matsuoka, K. Miuchi, T. Mizumoto, H. Nishimura, T. Oku, T. Sawano, T. Shinohara, J. Suzuki, A. Takada, T. Tanimori, K. Ueno, Spatial resolution of a $\mu$ PIC-based neutron imaging detectr, Nuclear Instruments and Methods in Physics Research Section A 726 (2013) 155 161. doi:10.1016/j.nima.2013.06.001

[37] R. Veenhof, Garfield - simulation of gaseous detectors URL http://garfield.web.cern.ch/garfield/

[38] S. Mukhopadhay, N. Majumdar, A nearly exact Boundary Element Method URL http://nebem.web.cern.ch/nebem/

[39] T. Mizumoto, Y. Matsuoka, Y. Mizumura, T. Tanimori, H. Kubo, A. Takada, S. Iwaki, T. Sawano, K. Nakamura, S. Komura, S. Nakamura, T. Kishimoto, M. Oda, S. Miyamoto, T. Takemura, J. Parker, D. Tomono, S. Sonoda, K. Miuchi, S. Kurosawa, New readout and data-acquisition system in an electron-tracking Compton camera for $\mathrm{MeV}$ gamma-ray astronomy (SMILE-II), Nuclear Instrument and Methods in Physics Research Section A 800 (2015) 40 - 50. doi:10.1016/j.nima. 2015.08.004

[40] T. Uchida, Hardware-Based TCP Processor for Gigabit Ethernet, IEEE Transactions on Nuclear Science 55 (2008) 3. doi:10.1109/TNS.2008.920264

[41] H. Baba, T. Ichihara, T. Ohnishi, S. Takeuchi, K. Yoshida, Y. Watanabe, S. Ota, S. Shimoura, New data acquisition system for the RIKEN Radioactive Isotope Beam Factory, Nuclear Instruments and Methods in Physics Research Section A 616 (2010) 65 - 68. doi:10.1016/j.nima.2010.02.120

[42] 3M, Enhanced Specular Refrector (ESR)

URL http://multimedia.3m.com/mws/media/3747300/ vikuiti-tm-esr-sales-literature.pdf?fn=ESR\%20ss2.pdf

[43] J. F. Ziegler, M. Ziegler, J. Biersack, SRIM - The stopping and range of ions in matter (2010), Nuclear Instruments and Methods in Physics Research Section B 268 (2010) 1818 - 1823. doi:10.1016/j.nimb.2010.02.091

[44] K. A. Olive, et al. (Particle Data Group), Physica Review C 38 (2014) 090001.

[45] S. Biagi, Magboltz - transport of electrons in gas mixtures URL http://magboltz.web.cern.ch/magboltz/

[46] P. Hough, Machine Analysis of Bubble Chamber Pictures, 2nd International Conference on High-Energy Accelerators and Instrumentation (1959) $554-558$.

[47] R. O. Duda, P. E. Hart, Use of the Hough transformation to detect lines and curves in pictures, Communications of the ACM 15 (1972) $11-15$. doi:10.1145/361237.361242

[48] T. Roger, M. Caaman̄o, C. Demonchy, W. Mittig, H. Savajols, I. Tanihata, Tracking algorithms for the active target MAYA, Nuclear Instruments and Methods in Physics Research Section A 638 (2011) 134 - 142. doi:10.1016/j.nima.2011.02.061

[49] Y. Ayyad, W. Mittig, D. Bazin, S. Beceiro-Novo, M. Cortesi, Novel particle tracking algorithm based on the Random Sample Consensus Model for the Active Target Time Projection Chamber (AT-TPC), Nuclear Instruments and Methods in Physics Research Section A 880 (2018) 166 - 173. doi:10.1016/j.nima. 2017.10 .090 